\title{
OPEN Effects of coalescence and isospin symmetry on the freezeout of light nuclei and their anti-particles
}

\author{
M. Waqas ${ }^{1}$, G. X. Peng ${ }^{1,2,3 凶}$, Fu-Hu Liu ${ }^{4,5}$ \& Z. Wazir
}

The transverse momentum spectra of light nuclei (deuteron, triton and helion) produced in various centrality intervals in Gold-Gold ( $\mathrm{Au}-\mathrm{Au})$, Lead-Lead ( $\mathrm{Pb}-\mathrm{Pb}$ ) and proton-Lead $(\mathrm{p}-\mathrm{Pb}$ ) collisions, as well as in inelastic (INEL) proton-proton $(p-p)$ collisions are analyzed by the blast wave model with Boltzmann Gibbs statistics. The model results are nearly in agreement with the experimental data measured by STAR and ALICE Collaborations in special transverse momentum ranges. We extracted the bulk properties in terms of kinetic freezeout temperature, transverse flow velocity and freezeout volume. It is observed that deuteron and anti-deuteron freezeout later than triton and helion as well as their anti-particles due to its smaller mass, while helion and triton, and anti-helion and anti-triton freezeout at the same time due to isospin symmetry at higher energies. It is also observed that light nuclei freezeout earlier than their anti-nuclei due to the large coalescence of nucleons for light nuclei compared to their anti-nuclei. The kinetic freezeout temperature, transverse flow velocity and kinetic freezeout volume decrease from central to peripheral collisions. Furthermore, the transverse flow velocity depends on mass of the particle which decreases with increasing the mass of the particle.

A new form of matter, the so called quark-gluon plasma (QGP) is produced at high temperatures and energy densities in relativistic heavy ion collisions. In nuclear physics, among the various probes, charmonia (i.e. $j / \psi$ suppression) are very sensitive probes of the characteristics of QGP ${ }^{1,2}$. Charmonia are the bound states of charm-anticharm $(c \bar{c})$ quarks, and they are formed early in the heavy ion collisions and their yields are expected to be suppressed in the medium. Numerous theoretical and experimental studies ${ }^{3-5}$ enrich our understanding of quarkonia as probes of QGP. The $j / \psi$ suppression was measured at SPS ${ }^{6,7}$ and was termed as 'anomalous' $j / \psi$ suppression and it was considered a hint for QGP. This matter is formed in the early stage of collisions that survives for a very short period of time $(\sim 7-10 \mathrm{fm} / \mathrm{c})$, after which the QGP gets transformed rapidly to a system of hadron gas. Due to multi-partonic interactions throughout the evolution time in the collisions, the information about the initial condition of the system get mostly lost. The final state behavior of such colliding system can be attained from the measurement of the number as well as the identity of the produced particles along with their energy and momentum spectra. The final state information are very useful to understand the particle production mechanisms and the nature of the matter created in these high energy collisions.

Temperature is one of the most crucial factor in sub-atomic physics. There are different types of temperatures present in literature $\mathrm{e}^{7-12}$. Chemical freezeout temperature $\left(T_{c h}\right)$, which describes the excitation degree of interacting system at the stage of chemical freezeout. At chemical freezeout the chemical components (relative fraction) of the particles are invariant. The excitation degree of the interacting system at the stage of thermal or kinetic freezeout is described by the kinetic freezeout temperature $\left(T_{0}\right)$. At kinetic freezeout, the transverse momentum spectra of the particles are no longer changed. Another type of temperature is the effective $\left(T_{\text {eff }}\right)$. It is not a real temperature but is related to the particle mass and can be extracted from the transverse momentum spectra by using various distribution laws such as standard (Boltzmann, Bose-Einstein and Fermi-Dirac), Tsallis, and so forth.

The chemical freezeout temperature is usually obtained from the particle ratio ${ }^{13-15}$. Due to chemical equilibrium being meanwhile or earlier than the kinetic equilibrium, the chemical freezeout temperature is equal

\footnotetext{
${ }^{1}$ School of Nuclear Science and Technology, University of Chinese Academy of Sciences, Beijing 100049, China. ${ }^{2}$ Theoretical Physics Center for Science Facilities, Institute of High Energy Physics, Beijing 100049, China. ${ }^{3}$ Synergetic Innovation Center for Quantum Effects \& Application, Hunan Normal University, Changsha 410081, China. ${ }^{4}$ Institute of Theoretical Physics \& State Key Laboratory of Quantum Optics and Quantum Optics Devices, Shanxi University, Taiyuan 030006, China. ${ }^{5}$ Collaborative Innovation Center of Extreme Optics, Shanxi University, Taiyuan 030006, China. ${ }^{6}$ Department of Physics, Ghazi University, Dera Ghazi Khan, Pakistan. ${ }^{凶}$ email: gxpeng@ucas.ac.cn
} 
or higher than the kinetic freezeout temperature. The effective temperature, due to mass and flow velocity are also larger than the kinetic freezeout temperature ${ }^{16,17}$. Due to more violent interactions in central collisions, both the chemical freezeout temperature and effective temperature are larger in central collisions than in the peripheral collisions. However, the situation for kinetic freezeout temperature is not clear. several literatures claim larger $T_{0}$ in central collisions ${ }^{18-23}$ which decrease towards periphery, while several claims larger $T_{0}$ in peripheral collisions ${ }^{24-27}$ which decrease towards the central collisions. In addition, volume is also very important parameter in high energy collisions. The volume occupied by the ejectiles when the correlative interactions become negligable and the only force they experience is Coulombic force, is said to be kinetic freezeout volume $(V)$. Most of the literatures agreed to the larger $V$ as well as the transverse flow velocity $\left(\beta_{T}\right)$ in central collisions which decrease towards periphery.

Freezeout scenario is very important in high energy collisions. Different freezeout scenarios are discussed in literature at different stages of the freezeout, but we will focus on kinetic freezeout scenarios in the present work. There are several kinetic freezeout scenarios in literature $22,23,28-32$ which include single, double, triple and multiple freezeout scenario. In the study of production of light nuclei, it is expected that the freezeout of the particles may also be dependent on the nucleon coalescence and isospin symmetry at higher energies. However it is hard to say that the coalescence and isospin symmetry play a role in $j / \psi$ suppression because its production mechanism is very complicated.

The transverse momentum spectra $\left(p_{T}\right)$ of the particles are very important observables because they give very crucial information about the equilibrium dynamics and the anisotropy of the produced system in heavy ion collisions ${ }^{31}$. In the present work, we will analyze the $p_{T}$ spectra of deuteron $(d)$, anti-deuteron $(\bar{d})$, triton $(t)$, anti-triton $(\bar{t})$, helion $\left({ }^{3} \mathrm{He}\right)$ and anti-helion $\left({ }^{3} \mathrm{He}\right)$ in Gold-Gold $(\mathrm{Au}-\mathrm{Au})$, Lead-Lead $(\mathrm{Pb}-\mathrm{Pb})$, Proton-Lead $(\mathrm{p}-\mathrm{Pb})$ and proton-proton $(\mathrm{p}-\mathrm{p})$ collisions.

\section{The method and formalism}

A few methods can be used for the extraction of $T_{0}$ and $\beta_{T}$, including but not limited to, (1) the blast-wave model with Boltzmann-Gibbs statistics $(\mathrm{BGBW})^{33-35}$, (2) the blast-wave model with Tsallis statistics ${ }^{36}$, (3) an alternative method using the Boltzmann distribution ${ }^{29,35-42}$, and (4) the alternative method using the Tsallis distribution ${ }^{43,44}$. It is noteworthy that $T_{0}$ is the intercept in the linear relation $T-m_{0}$ in alternative method, where $m_{0}$ is the rest mass; and $\beta_{T}$ is the slope in the linear relation $<p_{T}>-\bar{m}$, where $<p_{T}>$ is the mean transverse momentum and $\bar{m}$ is the mean moving mass (i.e., the mean energy).

Reference ${ }^{45}$ confirms that the above methods are harmonious. Among these methods, the first method is the most direct with fewer parameters, though it has been revised in various ways and applied to other quantities ${ }^{46-50}$. We have used the first method, i.e., BGBW in the present work. Due to their coherence, other models will not be used ${ }^{45}$.

BGBW is a phenomenological model which is used for the spectra of hadrons based on flowing local thermal sources with global variables of temperature, volume and transverse flow velocity. According to ${ }^{33-35}$, the $p_{T}$ distribution of BGBW can be written as

$$
\begin{aligned}
f\left(p_{T}\right)= & \frac{1}{N} \frac{d N}{d p_{T}}=\frac{1}{N} \frac{g V}{(2 \pi)^{2}} p_{T} m_{T} \int_{0}^{R} r d r \\
& \times I_{0}\left[\frac{p_{T} \sinh (\rho)}{T}\right] K_{1}\left[\frac{m_{T} \cosh (\rho)}{T}\right],
\end{aligned}
$$

where $N$ is the number of particles, $g$ represents the degeneracy factor of the particle (which is different for different particles, based on $g_{n}=2 S_{n}+1$, while $S_{n}$ is the spin of the particle), $V$ is the freezeout volume, $m_{T}$ is the transverse mass $\left(m_{T}=\sqrt{p_{T}^{2}+m_{0}^{2}}\right), I_{0}$ and $K_{1}$ are the modified Bessel functions, $\rho=\tanh ^{-1}[\beta(r)]$, $\beta(r)=\beta_{S}(r / R)^{n_{0}}$ is the transverse radial flow of the thermal source at radius $0 \leq r \leq R$ with surface velocity $\beta_{S}$ and $n_{0}=1^{36}$. In general, $\beta_{T}=\left(2 / R^{2}\right) \int_{0}^{R} r \beta(r) d r=2 \beta_{S} /\left(n_{0}+2\right)=2 \beta_{S} / 3$.

Equation (1) can be used for the fitting of $p_{T}$ spectra to obtain the parameters $T_{0}, V$ and $\beta_{T}$. It should be noted that Eq. (1) can be only valid in a narrow $p_{T}$ range i.e: they describe only soft excitation process. However we have to consider the hard scattering process for the spectra in a wide $p_{T}$ range. In general, the contribution of hard process can be parameterized to an inverse power law [51-453], i.e., Hagedorn function ${ }^{54,55}$

$$
f_{0}\left(p_{T}\right)=\frac{1}{N} \frac{d N}{d p_{T}}=A p_{T}\left(1+\frac{p_{T}}{p_{0}}\right)^{-n},
$$

which is resulted from the calculus of quantum chromodynamics (QCD) $)^{51-53}$, where $p_{0}$ and $n$ are the free parameters and $A$ is the normalization constant that depends on $p_{0}$ and $n$.

In order to describe a wide $p_{T}$ range, the superposition of both the soft and hard process can be used, which is

$$
f_{0}\left(p_{T}\right)=k f_{S}\left(p_{T}\right)+(1-k) f_{H}\left(p_{T}\right)
$$

where $k$ shows the contribution fraction of the first component (soft excitation), while $(1-k)$ represents the contribution fraction of the second component (hard scattering) in Eq. (3), and according to Hagedorn model ${ }^{55}$ the usual step function can be also be used for the superposition of soft and hard components.

According to Hagedorn thermal model ${ }^{55}$, the two-component BGBW distribution function can also be structured by using the usual step function, 

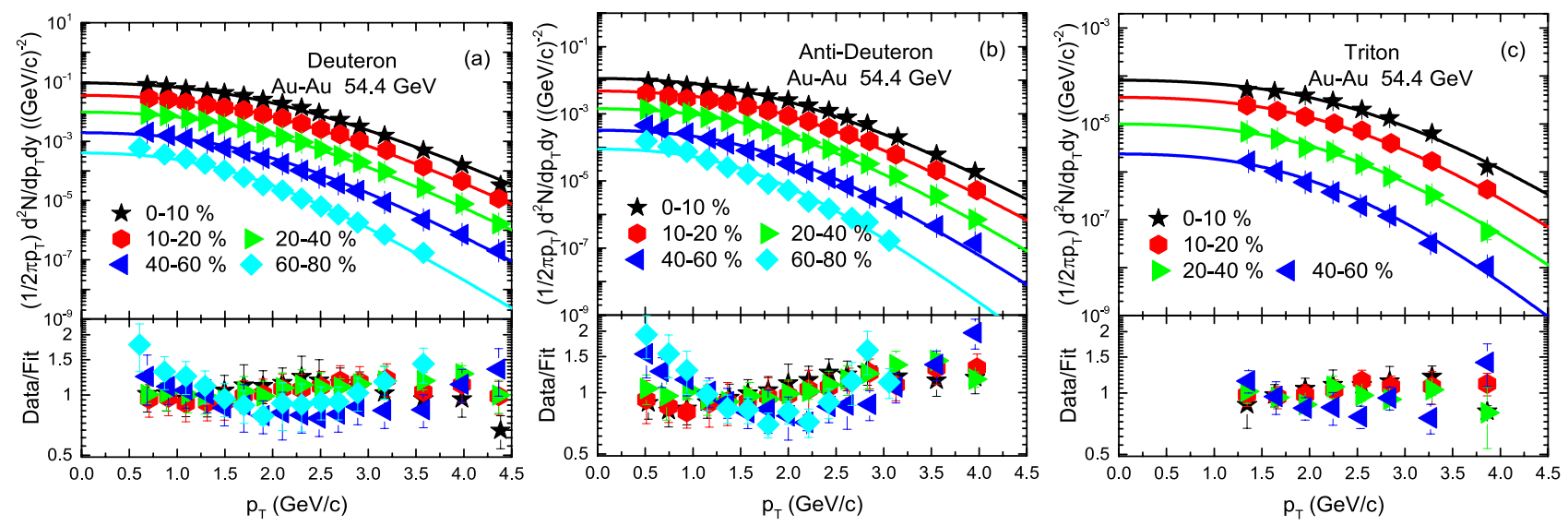

Figure 1. The transverse momentum $\left(p_{T}\right)$ spectra of (a) deutron, $(\mathbf{b})$ anti-deutron and (c) triteron produced in $|y|<0.5$ in different centrality classes in Au-Au collisions at $54.4 \mathrm{GeV}$. The symbols are the experimental data of the STAR Collaboration ${ }^{56}$ and the curves are our fitting by using BGBW statistics. Each panel is followed by the ratio of the data/fit.

$$
\begin{aligned}
f_{0}\left(p_{T}\right)= & \frac{1}{N} \frac{d N}{d p_{T}}=A_{1} \theta\left(p_{1}-p_{T}\right) f\left(p_{T}\right) \\
& +A_{2} \theta\left(p_{T}-p_{1}\right) f\left(p_{T}\right),
\end{aligned}
$$

where $A_{1}$ and $A_{2}$ are the constants which give the two components to be equal to each other at $p_{T}=p_{1}$.

\section{Results and discussion}

The transverse momentum $\left(p_{T}\right)$ spectra, $\left(1 / 2 \pi p_{T}\right) d^{2} N / d p_{T} d y$, of $d, \bar{d}$ and $t$ produced in AuAu collisions at $54.4 \mathrm{GeV}$ are analyzed by BGBW statistics in different centrality classes are demonstrated in Fig. 1 . The symbols represent the experimental data of the STAR Collaboration measured in the mid-rapidity range $|y|<0.5$ and the solid curve are the results of our fitting by using Eq. (1). The well approximate description of the model result to the experimental data of the STAR Collaboration ${ }^{56}$ in the special $p_{T}$ range can be seen. The event centralities and the values of free parameters are listed in Table 1. The free parameters include kinetic freezeout temperature $\left(T_{0}\right)$, transverse flow velocity $\left(\beta_{T}\right)$, kinetic freezeout volume $(V)$, normalization constant $\left(N_{0}\right), \chi^{2}$ and the degree of freedom (dof). Each panel is followed by the results of its corresponding ratio of the data/ fit. Figure 2 demonstrates the $p_{T}$ spectra, $\left(1 / N_{e v}\right) d^{2} N / d p_{T} d y$ of $d, \bar{d},{ }^{3} \mathrm{He}$ and ${ }^{3} \mathrm{He}$ in various centrality classes in $\mathrm{p}-\mathrm{Pb}$ collisions at $5.02 \mathrm{TeV}$. The symbols stands for the experimental data of the ALICE Collaboration ${ }^{57}$ by the Large Hadron Collider (LHC) and the solid curve represent our fitting results by using the BGBW statistics. In Fig. 2 some spectra are scaled; such as the spectra of $d$ and $\bar{d}$ in centrality intervals $5-10 \%, 10-20 \%, 20-30 \%$, $30-40 \%, 40-50 \%, 50-60 \%, 60-70 \%, 70-80 \%$ and $90-90 \%$ are multiplied by $1 / 2,1 / 4,1 / 8,1 / 16,1 / 30,1 / 40,1 / 40$, $1 / 60$ and $1 / 60$, respectively.

Figure 3 is similar to Fig. 2, but it shows the $p_{T}$ spectra of $d, \bar{d}$ and $\left({ }^{3} \mathrm{He}+{ }^{3} \overline{\mathrm{He}}\right) / 2$ produced in different centrality intervals in $\mathrm{p}-\mathrm{Pb}$ collisions at $5.02 \mathrm{TeV}$. The symbols show the experimental data measured by the ALICE Collaboration in the rapidity region $-1 \leq y \leq 1$ and -1 y 0 respectively, and the curves are the results of our fitting by using Eq. (1). The well approximate description of the model results to the experimental data of the ALICE Collaboration ${ }^{58,59}$ in the special $p_{T}$ range can be seen.

In Fig. 4 the $p_{T}$ spectra of $d, d, t, \bar{t},{ }^{3} \mathrm{He}$ and ${ }^{3} \mathrm{He}$ in inelastic (INEL) $\mathrm{p}-\mathrm{p}$ collisions at $7 \mathrm{TeV}$ are presented. The symbols represent the experimental data of the ALICE Collaboration by the LHC in the rapidity interval of $|y|<0.5$ and the results of our fitting is represented by curve. The spectra of $\bar{d}, t, \bar{t},{ }^{3} \mathrm{He}$ and ${ }^{3} \mathrm{He}$ are multiplied by $1 / 2.5,800,400,100$ and 50 , respectively. One can see the well approximate description of the model results to the experimental data of the ALICE Collaboration ${ }^{60}$ in the special $p_{T}$ range.

Figure 5 shows the variation trend of parameters with centrality (mass). Panels a-d show the results for $\mathrm{Au}-\mathrm{Au}$ collisions at $54.4 \mathrm{GeV}, \mathrm{Pb}-\mathrm{Pb}$ collisions at $5.02 \mathrm{TeV}, \mathrm{p}-\mathrm{Pb}$ collisions at $5.02 \mathrm{TeV}$ and $\mathrm{p}-\mathrm{p}$ collisions at $7 \mathrm{TeV}$ respectively. Panels a-c show the dependence of $T_{0}$ on centrality, and panel $\mathrm{d}$ shows the dependence of $T_{0}$ on $m_{0}$. The types of particles are represented by different symbols. In Fig. 5, panels a-c, one can see that $d$, $\bar{d}, t, \bar{t},{ }^{3} \mathrm{He}$ and ${ }^{3} \mathrm{He}$ in all collisions ( $\mathrm{Au}-\mathrm{Au}, \mathrm{Pb}-\mathrm{Pb}$ and $\mathrm{p}-\mathrm{Pb}$ ) results in larger $T_{0}$ in central collisions which decrease towards periphery. The reason behind this is, in central collisions, large number of participants involve in interaction and the collisions are more violent that results in higher degree of excitation of the system and the kinetic freezeout temperature is high. However, the collisions become less violent as the centrality decreases and less number of participants involve in the interactions which results in comparatively low kinetic freezeout temperature. This is in agreement with Refs. ${ }^{18-23}$, but in disagreement with Refs. ${ }^{24-27}$.

Figure 5 includes Panel a $d, \bar{d}$, and $t$; Panel b $d, \bar{d}$, $t$ and $\bar{t}$; Panel $\mathrm{c} d, \bar{d}$ and $\left({ }^{3} \mathrm{He}+{ }^{3} \overline{\mathrm{He}}\right) / 2$; and Panel $\mathrm{d} d, \bar{d}$, $t, \bar{t},{ }^{3} \mathrm{He}$ and ${ }^{3} \mathrm{He}$. In some panels some particles and their anti-particles are missing due to the unavailability of data. In panel a one can see that $T_{0}$ is larger for $t$ than both of $d$ and $\bar{d}$ due to its mass. While $d$ and $\bar{d}$ has the same mass but $d$ freezeout earlier than $\bar{d}$. Similarly in panel $\mathrm{b} d, \bar{d},{ }^{3} \mathrm{He}$ and ${ }^{3} \mathrm{He}$, the mass of ${ }^{3} \mathrm{He}$ and ${ }^{3} \mathrm{He}$ is 


\begin{tabular}{|c|c|c|c|c|c|c|c|}
\hline Collisions & Centrality & Particle & $T_{0}(\mathrm{GeV})$ & $\beta_{T}(c)$ & $V\left(f m^{3}\right)$ & $N_{0}$ & $\chi^{2} /$ dof \\
\hline \multirow{5}{*}{$\begin{array}{l}\text { Figure } 1 \\
\mathrm{Au}-\mathrm{Au} \\
54.4 \mathrm{GeV}\end{array}$} & $0-10 \%$ & $d$ & $0.118 \pm 0.006$ & $0.460 \pm 0.009$ & $3500 \pm 183$ & $4.5 \times 10^{-4} \pm 5 \times 10^{-5}$ & $10 / 11$ \\
\hline & $10-20 \%$ & - & $0.113 \pm 0.006$ & $0.452 \pm 0.008$ & $3350 \pm 176$ & $1.7 \times 10^{-4} \pm 5 \times 10^{-5}$ & $4 / 11$ \\
\hline & $20-40 \%$ & - & $0.109 \pm 0.005$ & $0.440 \pm 0.007$ & $3200 \pm 150$ & $4 \times 10^{-5} \pm 5 \times 10^{-6}$ & $11 / 11$ \\
\hline & $40-60 \%$ & - & $0.105 \pm 0.005$ & $0.427 \pm 0.009$ & $3000 \pm 207$ & $9 \times 10^{-6} \pm 5 \times 10^{-7}$ & $14 / 11$ \\
\hline & $60-80 \%$ & - & $0.101 \pm 0.006$ & $0.388 \pm 0.010$ & $2800 \pm 210$ & $1.6 \times 10^{-6} \pm 6 \times 10^{-7}$ & $17 / 9$ \\
\hline \multirow{5}{*}{$\begin{array}{l}\mathrm{Au}-\mathrm{Au} \\
54.4 \mathrm{GeV}\end{array}$} & $0-10 \%$ & $\bar{d}$ & $0.110 \pm 0.005$ & $0.460 \pm 0.011$ & $3200 \pm 170$ & $5.9 \times 10^{-5} \pm 4 \times 10^{-6}$ & $12 / 10$ \\
\hline & $10-20 \%$ & - & $0.105 \pm 0.006$ & $0.453 \pm 0.010$ & $3100 \pm 180$ & $2.45 \times 10^{-5} \pm 5 \times 10^{-6}$ & $10 / 10$ \\
\hline & $20-40 \%$ & - & $0.100 \pm 0.005$ & $0.440 \pm 0.008$ & $2900 \pm 180$ & $7.2 \times 10^{-6} \pm 6 \times 10^{-7}$ & $22 / 10$ \\
\hline & $40-60 \%$ & - & $0.097 \pm 0.006$ & $0.427 \pm 0.011$ & $2760 \pm 170$ & $1.6 \times 10^{-6} \pm 4 \times 10^{-7}$ & $44 / 10$ \\
\hline & $60-80 \%$ & - & $0.092 \pm 0.006$ & $0.388 \pm 0.012$ & $2650 \pm 140$ & $3.7 \times 10^{-7} \pm 5 \times 10^{-8}$ & $34 / 8$ \\
\hline \multirow{4}{*}{$\begin{array}{l}\mathrm{Au}-\mathrm{Au} \\
54.4 \mathrm{GeV}\end{array}$} & $0-10 \%$ & $t$ & $0.130 \pm 0.005$ & $0.420 \pm 0.010$ & $2700 \pm 190$ & $1.3 \times 10^{-6} \pm 6 \times 10^{-7}$ & $16 / 3$ \\
\hline & $10-20 \%$ & - & $0.126 \pm 0.005$ & $0.400 \pm 0.012$ & $2500 \pm 150$ & $5.5 \times 10^{-7} \pm 4 \times 10^{-8}$ & $2 / 3$ \\
\hline & $20-40 \%$ & - & $0.122 \pm 0.006$ & $0.388 \pm 0.009$ & $2360 \pm 128$ & $1.5 \times 10^{-7} \pm 3 \times 10^{-8}$ & $8 / 3$ \\
\hline & $40-60 \%$ & - & $0.118 \pm 0.005$ & $0.363 \pm 0.012$ & $2200 \pm 146$ & $3.3 \times 10^{-8} \pm 5 \times 10^{-9}$ & $16 / 3$ \\
\hline \multirow{10}{*}{$\begin{array}{l}\text { Figure } 2 \\
\mathrm{~Pb}-\mathrm{Pb} \\
5.02 \mathrm{TeV}\end{array}$} & $0-5 \%$ & $d$ & $0.156 \pm 0.005$ & $0.545 \pm 0.009$ & $5100 \pm 172$ & $0.077 \pm 0.004$ & $1 / 14$ \\
\hline & $5-10 \%$ & - & $0.153 \pm 0.004$ & $0.540 \pm 0.012$ & $4940 \pm 160$ & $0.037 \pm 0.005$ & $3 / 14$ \\
\hline & $10-20 \%$ & - & $0.150 \pm 0.005$ & $0.535 \pm 0.012$ & $4710 \pm 160$ & $0.015 \pm 0.004$ & $4 / 14$ \\
\hline & $20-30 \%$ & - & $0.147 \pm 0.005$ & $0.527 \pm 0.009$ & $4500 \pm 167$ & $0.006 \pm 0.0004$ & $5 / 14$ \\
\hline & $30-40 \%$ & - & $0.145 \pm 0.005$ & $0.510 \pm 0.009$ & $4300 \pm 167$ & $0.0023 \pm 0.0004$ & $6 / 14$ \\
\hline & $40-50 \%$ & - & $0.143 \pm 0.005$ & $0.500 \pm 0.009$ & $4200 \pm 167$ & $7.5 \times 10^{-4} \pm 3 \times 10^{-5}$ & $6 / 14$ \\
\hline & $50-60 \%$ & - & $0.140 \pm 0.005$ & $0.480 \pm 0.012$ & $4100 \pm 200$ & $2.3 \times 10^{-4} \pm 3 \times 10^{-5}$ & $21 / 14$ \\
\hline & 60-70\% & - & $0.137 \pm 0.006$ & $0.450 \pm 0.010$ & $3910 \pm 190$ & $6.3 \times 10^{-5} \pm 4 \times 10^{-6}$ & $29 / 11$ \\
\hline & $70-80 \%$ & - & $0.133 \pm 0.005$ & $0.440 \pm 0.012$ & $3500 \pm 160$ & $2.3 \times 10^{-5} \pm 3 \times 10^{-6}$ & $16 / 9$ \\
\hline & $80-90 \%$ & - & $0.130 \pm 0.006$ & $0.420 \pm 0.010$ & $3300 \pm 150$ & $3 \times 10^{-6} \pm 5 \times 10^{-7}$ & $12 / 6$ \\
\hline \multirow{10}{*}{$\begin{array}{l}\mathrm{Pb}-\mathrm{Pb} \\
5.02 \mathrm{TeV}\end{array}$} & $0-5 \%$ & $\bar{d}$ & $0.148 \pm 0.005$ & $0.545 \pm 0.009$ & $4700 \pm 181$ & $0.086 \pm 0.005$ & $3 / 14$ \\
\hline & $5-10 \%$ & - & $0.145 \pm 0.006$ & $0.540 \pm 0.012$ & $4500 \pm 190$ & $0.04 \pm 0.006$ & $5 / 14$ \\
\hline & $10-20 \%$ & - & $0.142 \pm 0.005$ & $0.535 \pm 0.011$ & $4300 \pm 172$ & $0.0166 \pm 0.004$ & $5 / 14$ \\
\hline & $20-30 \%$ & - & $0.140 \pm 0.005$ & $0.527 \pm 0.012$ & $4100 \pm 150$ & $0.007 \pm 0.0004$ & $6 / 14$ \\
\hline & $30-40 \%$ & - & $0.137 \pm 0.006$ & $0.510 \pm 0.010$ & $3900 \pm 170$ & $0.0026 \pm 0.0004$ & $9 / 14$ \\
\hline & $40-50 \%$ & - & $0.133 \pm 0.005$ & $0.500 \pm 0.012$ & $3700 \pm 180$ & $8.5 \times 10^{-4} \pm 5 \times 10^{-5}$ & $12 / 14$ \\
\hline & $50-60 \%$ & - & $0.130 \pm 0.004$ & $0.480 \pm 0.010$ & $3500 \pm 156$ & $2.6 \times 10^{-4} \pm 4 \times 10^{-5}$ & $26 / 14$ \\
\hline & $60-70 \%$ & - & $0.128 \pm 0.005$ & $0.450 \pm 0.008$ & $3300 \pm 160$ & $7.2 \times 10^{-5} \pm 6 \times 10^{-6}$ & $22 / 11$ \\
\hline & $70-80 \%$ & - & $0.125 \pm 0.004$ & $0.440 \pm 0.010$ & $3100 \pm 180$ & $2.6 \times 10^{-5} \pm 5 \times 10^{-6}$ & $16 / 9$ \\
\hline & $80-90 \%$ & - & $0.122 \pm 0.005$ & $0.420 \pm 0.012$ & $2900 \pm 140$ & $3.2 \times 10^{-6} \pm 4 \times 10^{-7}$ & $15 / 6$ \\
\hline \multirow{3}{*}{$\begin{array}{l}\mathrm{Pb}-\mathrm{Pb} \\
5.02 \mathrm{TeV}\end{array}$} & $0-10 \%$ & ${ }^{3} \mathrm{He}$ & $0.164 \pm 0.005$ & $0.510 \pm 0.007$ & $4400 \pm 160$ & $1.5 \times 10^{-7} \pm 4 \times 10^{-8}$ & $3 / 1$ \\
\hline & $10-40 \%$ & - & $0.160 \pm 0.005$ & $0.500 \pm 0.008$ & $4200 \pm 140$ & $9 \times 10^{-8} \pm 4 \times 10^{-9}$ & $2 / 1$ \\
\hline & $40-100 \%$ & - & $0.156 \pm 0.004$ & $0.470 \pm 0.010$ & $4000 \pm 180$ & $4.7 \times 10^{-8} \pm 4 \times 10^{-9}$ & $10 / 1$ \\
\hline \multirow{3}{*}{$\begin{array}{l}\mathrm{Pb}-\mathrm{Pb} \\
5.02 \mathrm{TeV}\end{array}$} & $0-10 \%$ & ${ }^{3} \overline{\mathrm{He}}$ & $0.158 \pm 0.004$ & $0.510 \pm 0.007$ & $4000 \pm 140$ & $1.4 \times 10^{-7} \pm 4 \times 10^{-8}$ & $4 / 1$ \\
\hline & $10-40 \%$ & - & $0.154 \pm 0.005$ & $0.505 \pm 0.010$ & $3800 \pm 166$ & $9.5 \times 10^{-8} \pm 4 \times 10^{-8}$ & $3 / 1$ \\
\hline & $30-100 \%$ & - & $0.151 \pm 0.005$ & $0.475 \pm 0.009$ & $3600 \pm 155$ & $5.3 \times 10^{-8} \pm 4 \times 10^{-9}$ & $4 / 1$ \\
\hline \multirow{5}{*}{$\begin{array}{l}\text { Figure } 3 \\
\text { p-Pb } \\
5.02 \mathrm{TeV}\end{array}$} & $0-10 \%$ & $d$ & $0.148 \pm 0.006$ & $0.480 \pm 0.008$ & $4720 \pm 170$ & $1.25 \times 10^{-4} \pm 4 \times 10^{-5}$ & $12 / 7$ \\
\hline & $10-20 \%$ & - & $0.143 \pm 0.006$ & $0.470 \pm 0.011$ & $4550 \pm 160$ & $4.5 \times 10^{-5} \pm 5 \times 10^{-6}$ & $18 / 7$ \\
\hline & $20-40 \%$ & - & $0.139 \pm 0.006$ & $0.440 \pm 0.007$ & $4400 \pm 186$ & $1.7 \times 10^{-5} \pm 6 \times 10^{-6}$ & $13 / 4$ \\
\hline & $40-60 \%$ & - & $0.135 \pm 0.005$ & $0.430 \pm 0.008$ & $4200 \pm 179$ & $5 \times 10^{-6} \pm 5 \times 10^{-7}$ & $21 / 4$ \\
\hline & $60-100 \%$ & - & $0.131 \pm 0.005$ & $0.400 \pm 0.010$ & $4130 \pm 180$ & $8 \times 10^{-7} \pm 6 \times 10^{-8}$ & $35 / 4$ \\
\hline \multirow{5}{*}{$\begin{array}{l}\text { p-Pb } \\
5.02 \mathrm{TeV}\end{array}$} & $0-10 \%$ & $\bar{d}$ & $0.140 \pm 0.005$ & $0.480 \pm 0.012$ & $4400 \pm 184$ & $1.31 \times 10^{-4} \pm 6 \times 10^{-5}$ & $8 / 7$ \\
\hline & $10-20 \%$ & - & $0.136 \pm 0.006$ & $0.470 \pm 0.010$ & $4200 \pm 140$ & $4.4 \times 10^{-5} \pm 6 \times 10^{-6}$ & $18 / 5$ \\
\hline & $20-40 \%$ & - & $0.132 \pm 0.005$ & $0.440 \pm 0.009$ & $4000 \pm 160$ & $1.75 \times 10^{-5} \pm 7 \times 10^{-6}$ & $8 / 5$ \\
\hline & $40-60 \%$ & - & $0.128 \pm 0.004$ & $0.430 \pm 0.008$ & $3800 \pm 154$ & $5.5 \times 10^{-6} \pm 7 \times 10^{-7}$ & $21 / 4$ \\
\hline & $60-100 \%$ & - & $0.122 \pm 0.004$ & $0.400 \pm 0.010$ & $3600 \pm 168$ & $1 \times 10^{-6} \pm 4 \times 10^{-7}$ & $16 / 4$ \\
\hline & & & & & & & \\
\hline
\end{tabular}




\begin{tabular}{|c|c|c|c|c|c|c|c|}
\hline Collisions & Centrality & Particle & $T_{0}(\mathrm{GeV})$ & $\beta_{T}(c)$ & $V\left(f m^{3}\right)$ & $N_{0}$ & $\chi^{2} /$ dof \\
\hline \multirow{4}{*}{$\begin{array}{l}\text { p-Pb } \\
5.02 \mathrm{TeV}\end{array}$} & $0-10 \%$ & $\left({ }^{3} \mathrm{He}+{ }^{3} \overline{\mathrm{He}}\right) / 2$ & $0.154 \pm 0.006$ & $0.470 \pm 0.008$ & $4000 \pm 191$ & $5 \times 10^{-8} \pm 4 \times 10^{-9}$ & $4 /-$ \\
\hline & $10-20 \%$ & - & $0.150 \pm 0.005$ & $0.443 \pm 0.011$ & $3800 \pm 166$ & $1.4 \times 10^{-8} \pm 5 \times 10^{-9}$ & $3 /-$ \\
\hline & $20-40 \%$ & - & $0.146 \pm 0.005$ & $0.413 \pm 0.009$ & $3600 \pm 165$ & $5 \times 10^{-9} \pm 3 \times 10^{-10}$ & $26 /-$ \\
\hline & $40-100 \%$ & - & $0.142 \pm 0.005$ & $0.410 \pm 0.011$ & $3400 \pm 150$ & $7 \times 10^{-10} \pm 3 \times 10^{-11}$ & $1 /-$ \\
\hline \multirow{6}{*}{$\begin{array}{l}\text { Figure } 4 \\
\text { p-p } \\
7 \mathrm{TeV}\end{array}$} & - & $d$ & $0.090 \pm 0.006$ & $0.420 \pm 0.008$ & $3000 \pm 158$ & $2 \times 10^{-6} \pm 4 \times 10^{-7}$ & $141 / 17$ \\
\hline & - & $\bar{d}$ & $0.080 \pm 0.005$ & $0.420 \pm 0.007$ & $2600 \pm 145$ & $2 \times 10^{-6} \pm 4 \times 10^{-7}$ & $81 / 16$ \\
\hline & - & $t$ & $0.105 \pm 0.004$ & $0.350 \pm 0.009$ & $2000 \pm 155$ & $1.3 \times 10^{-10} \pm 3 \times 10^{-11}$ & $0.02 /-$ \\
\hline & - & $\bar{t}$ & $0.095 \pm 0.004$ & $0.350 \pm 0.008$ & $2000 \pm 145$ & $1.6 \times 10^{-10} \pm 4 \times 10^{-11}$ & $0.02 /-$ \\
\hline & - & ${ }^{3} \mathrm{He}$ & $0.105 \pm 0.004$ & $0.300 \pm 0.007$ & $2000 \pm 155$ & $1.3 \times 10^{-10} \pm 3 \times 10^{-11}$ & $0.6 /-$ \\
\hline & - & ${ }^{3} \overline{\mathrm{He}}$ & $0.095 \pm 0.005$ & $0.350 \pm 0.007$ & $2000 \pm 145$ & $6 \times 10^{11} \pm 4 \times 10^{-12}$ & $38 /-$ \\
\hline
\end{tabular}

Table 1. List of the parameters. - is used in some places instead of dof. In fact it is not the fit result. if dof $<0$, the we put - instead of negative values.
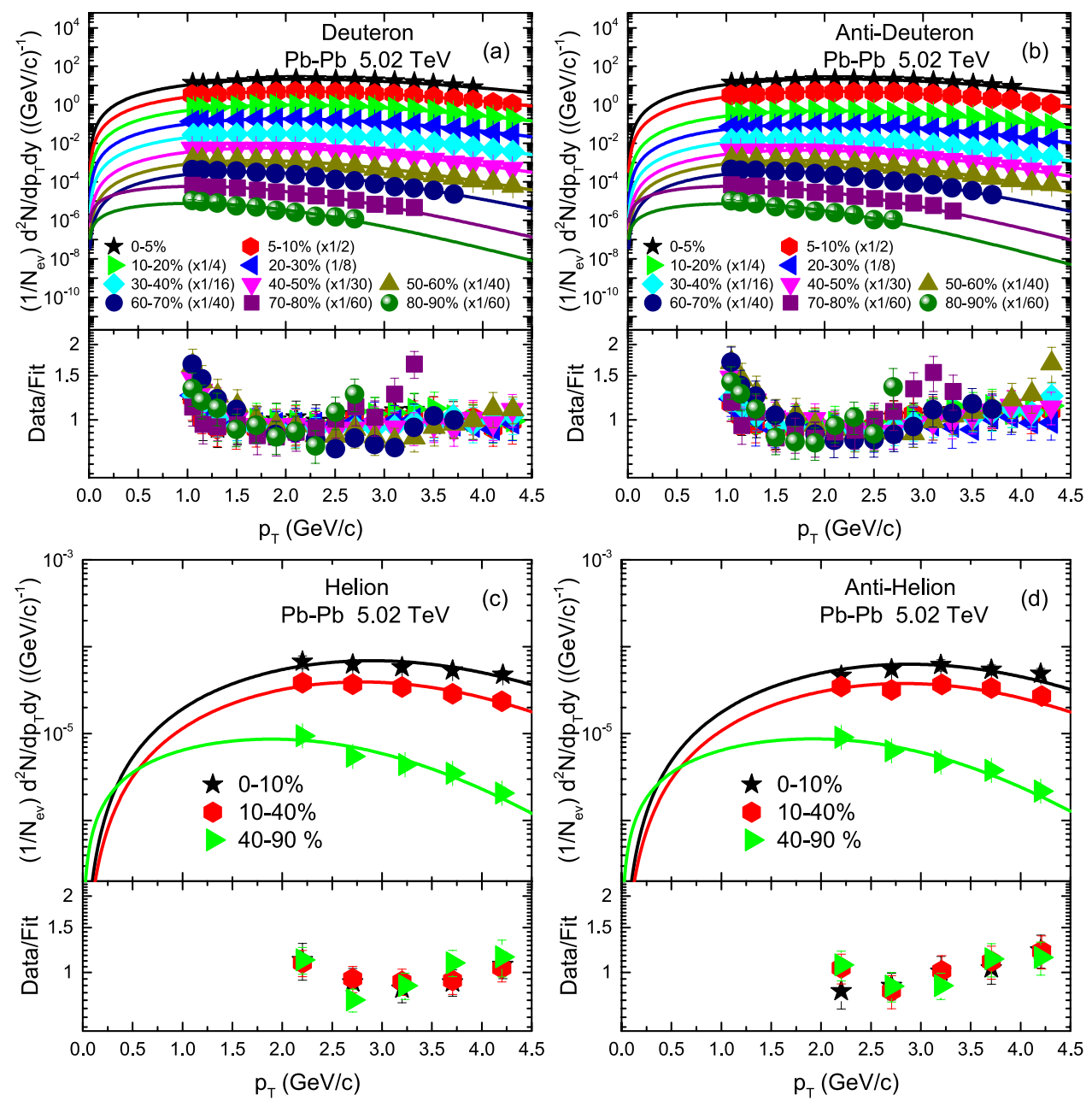

Figure 2. Transverse momentum spectra of $d, \bar{d},{ }^{3} \mathrm{He}$ and ${ }^{3} \mathrm{He}$ in $|y|<0.5$ produced in different centrality intervals in $\mathrm{Pb}-\mathrm{Pb}$ collisions at 5.02 TeV. The symbols represent the experimental data measured by the ALICE Collaboration $^{57}$, while the curves are our fitted results by using BGBW statistics, Eq. (1). Each panel is followed by the ratio of the data/fit. 

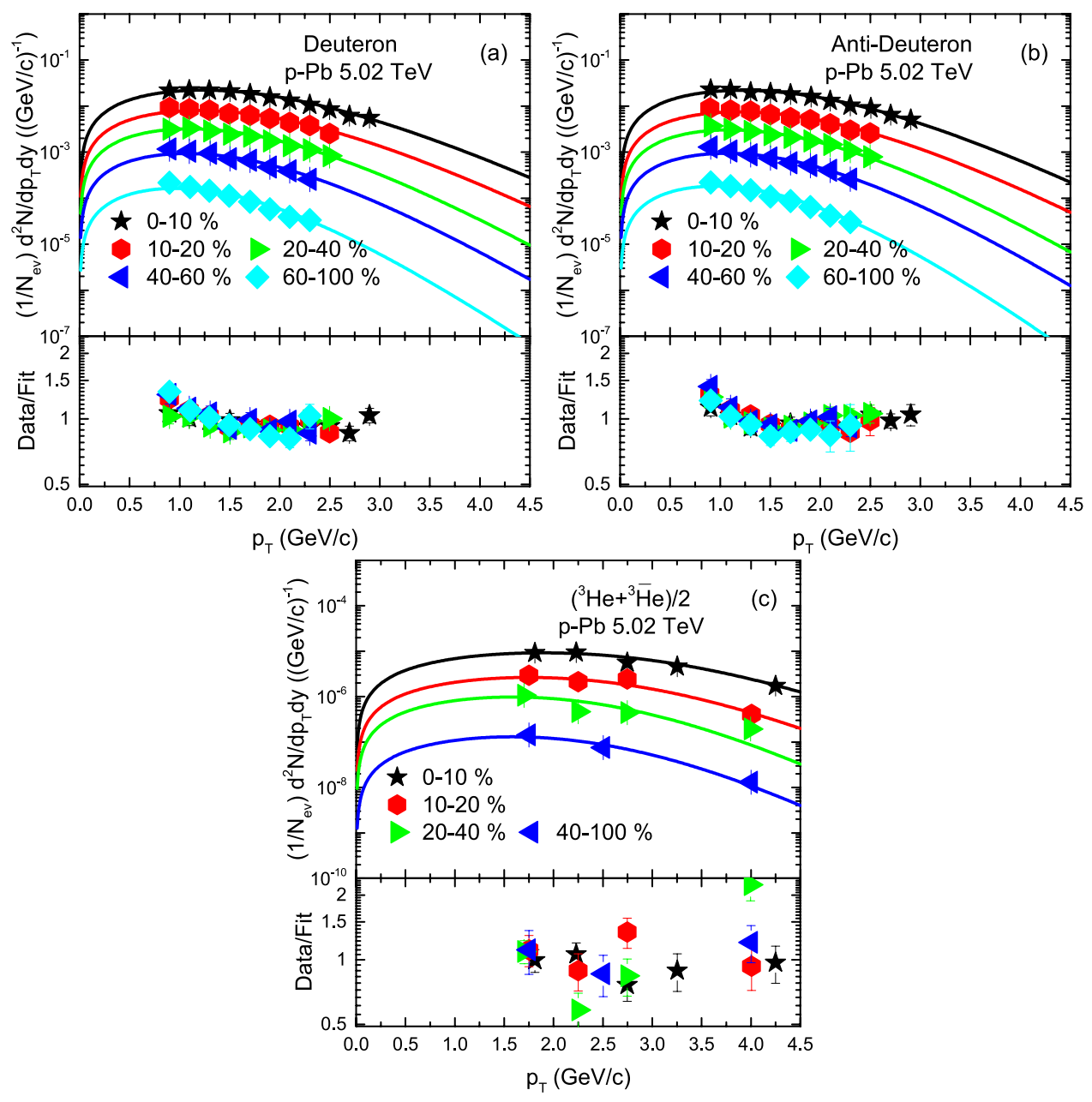

Figure 3. Transverse momentum spectra of $(\mathbf{a}) d,(\mathbf{b}) \bar{d}$ and $(\mathbf{c})\left({ }^{3} \mathrm{He}+{ }^{3} \overline{\mathrm{He}}\right) / 2$ produced in various centrality bins in $\mathrm{p}-\mathrm{Pb}$ collisions at $5.02 \mathrm{TeV}$. The symbols represent the experimental data measured by ALICE Collaboration $^{58,59}$, while the curves are our fitted results by using BGBW statistics, Eq. (1). Each panel is followed by the ratio of the data/fit.

larger than $d$ and $\bar{d}$, therefore they freezeout earlier than $d$ and $\bar{d}$, but $d$ and ${ }^{3}$ He freezeout earlier than $\bar{d}$ and ${ }^{3} \overline{\mathrm{He}}$ respectively, while in panel c $\left({ }^{3} \mathrm{He}+{ }^{3} \mathrm{He}\right) / 2$ has larger $T_{0}$ than $d$ and $\bar{d}$ whereas $d$ freezeout earlier than $\bar{d}$, and in Panel $\mathrm{d}^{3} \mathrm{He}$ and ${ }^{3} \mathrm{He}$, as well as $t$ and $\bar{t}$ freezeout earlier than $d$ and $\bar{d}$, and the values for ${ }^{3} \mathrm{He}$ and $t$, and ${ }^{3} \mathrm{He}$ and $\bar{t}$ are respectively the same. Basically, the formation of light nuclei occur by the coalescence of nucleons with similar momenta. In the present work, we believe that the coalescence of nucleons is larger for $d, t$ and ${ }^{3} \mathrm{He}$ compared to their anti-particles and therefore $T_{0}$ is larger for light nuclei than for their anti-nuclei. Furthermore, we observed that ${ }^{3} \mathrm{He}$, and $t$, and ${ }^{3} \mathrm{He}$ and $\bar{t}$ freezeout at the same time. In our opinion this is due to the isospin symmetry at high energies which occurs in nearly identical masses (e.g. triton and helion) where an up quark is replaced by a down quark. In addition, $T_{0}$ in $\mathrm{Pb}-\mathrm{Pb}$ is larger than in $\mathrm{Au}-\mathrm{Au}$ and in the later, it is larger than in $\mathrm{p}-\mathrm{p}$ collisions which shows its dependence on the cross-section of interacting system. However $T_{0}$ is larger in $\mathrm{p}-\mathrm{Pb}$ than in $\mathrm{Au}-\mathrm{Au}$ collisions because the center of mass energy for $\mathrm{p}-\mathrm{Pb}$ is $5.02 \mathrm{TeV}$ which is very larger than the center of mass energy of $\mathrm{Au}-\mathrm{Au}$ collision $(54.4 \mathrm{GeV})$, and this may reveal its dependence on energy.

Figure 6 is similar to Fig. 5, but shows the dependence of $\beta_{T}$ on centrality in panels a-c, while panel d shows its dependence on $m_{0}$. It can be seen that $\beta_{T}$ decrease from central to peripheral collisions due to large number 


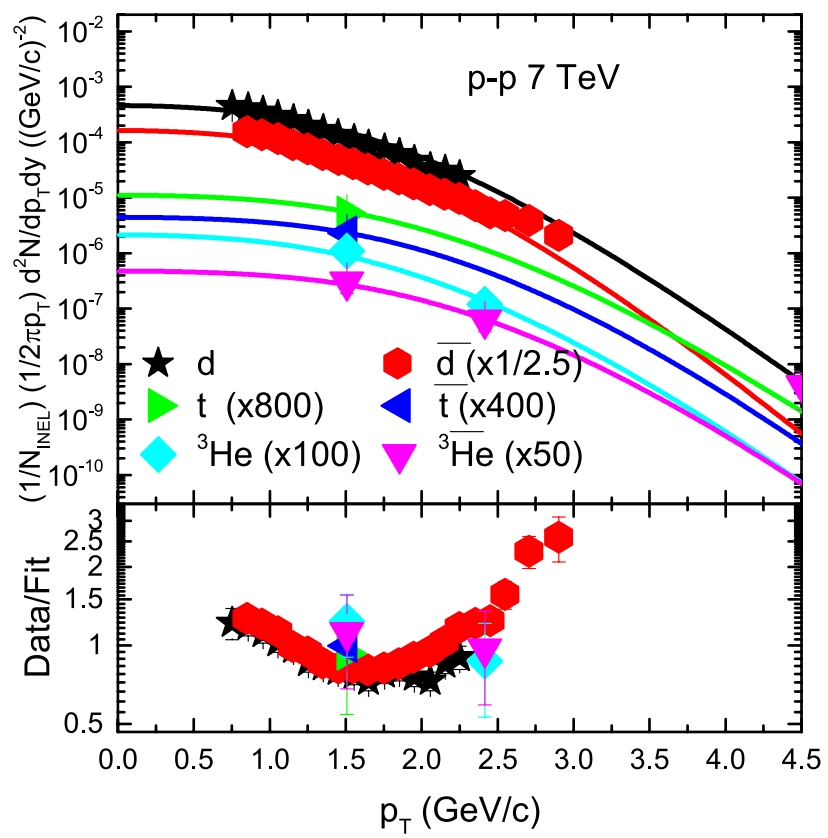

Figure 4. Figure 4 is similar to Figs. 2 and 3 , but it shows $p_{T}$ spectra of $d, \bar{d}, t, \bar{t},{ }^{3} \mathrm{He}$ and ${ }^{3} \mathrm{He}$ in $|y|<0.5$ produced in INEL p-p collisions at $7 \mathrm{TeV}$. The symbols represent the experimental data measured by the ALICE Collaboration $^{60}$, while the curves are our fitted results by using BGBW statistics, Eq. (1). Each panel is followed by the ratio of the data/fit.

of participants in central collision that experience more violent squeeze and results in a rapid expansion of the system. While this expansion becomes steady from central to periphery due to decreasing the participant nucleons which results in comparatively weak squeeze. Furthermore, $\beta_{T}$ is mass dependent. Greater the mass of the particle is, smaller the value of $\beta_{T}$. $\beta_{T}$ for nuclei and anti-nuclei is the same. Besides, $\beta_{T}$ shows dependence on the cross-section of interacting system. Larger the cross-section of interacting system, larger the $\beta_{T}$ is. However $\beta_{T}$ is slightly larger in $\mathrm{p}-\mathrm{Pb}$ collisions than in $\mathrm{Au}-\mathrm{Au}$ collisions due to the effect of very large center of mass energy of $\mathrm{p}-\mathrm{Pb}$ than $\mathrm{Au}-\mathrm{Au}$ collisions.

Figure 7 is similar to Fig. 6, but shows the dependence of $V$ on centrality (mass). It can be seen that $V$ decreases from central to peripheral collisions in panel a-c, as the number of participant nucleons decreases from central collisions to periphery depending on the interaction volume. The system with more participants reaches to equilibrium quickly due to large number of secondary collisions by the re-scattering of partons, which decreases towards periphery and the system goes away from equilibrium state. In addtion, $V$ for deuteron and anti-deuteron is larger than that of triton and anti-triton as well as from helion and anti-helion. The parameter $V$ for nuclei is larger than their anti-nuclei due to larger coalescence of nucleons for the nuclei than for their anti-nuclei. In case of triton and anti-triton and helion and anti-helion, $\mathrm{V}$ of triton and helion, and anti-triton and anti-helion are respectively the same. Besides, $V$ is larger in $\mathrm{Pb}-\mathrm{Pb}$ collisions that the rest, and in $\mathrm{Au}-\mathrm{Au}$ collisions as well as $\mathrm{p}-\mathrm{Pb}$ collisions it is larger than in $\mathrm{p}-\mathrm{p}$ collisions which shows the dependence of $V$ on the cross-section of interacting system. However $\mathrm{V}$ is larger in $\mathrm{p}-\mathrm{Pb}$ than in $\mathrm{Au}-\mathrm{Au}$ collisions. We think that this is due the effect of very higher center energy of $\mathrm{p}-\mathrm{Pb}$ collisions compared to $\mathrm{Au}-\mathrm{Au}$ collisions, because higher energy corresponds to longer evolution time which may lead to larger partonic system.

Figure $8 \mathrm{a}-\mathrm{c}$ show the dependence of $N_{0}$ on centrality. One can see that $N_{0}$ decrease with decreasing centrality. Furthermore, the parameter $N_{0}$ depends on mass of the particle. In panels a-d the parameter $N_{0}$ for deuteron and anti-deuteron are larger than triton, and in (a) it is larger for deuteron than anti-deuteron due to large coalescence of deuteron, while in panels b-d the parameter $N_{0}$ for nuclei and their anti-particles are the almost same. In general, the parameter $N_{0}$ is the same for nuclei and their anti-nuclei. The parameter $N_{0}$ for triton and helion, and anti-triton and anti-helion is the same due to the isopin symmetry. In deed $N_{0}$ is only a normalization factor 

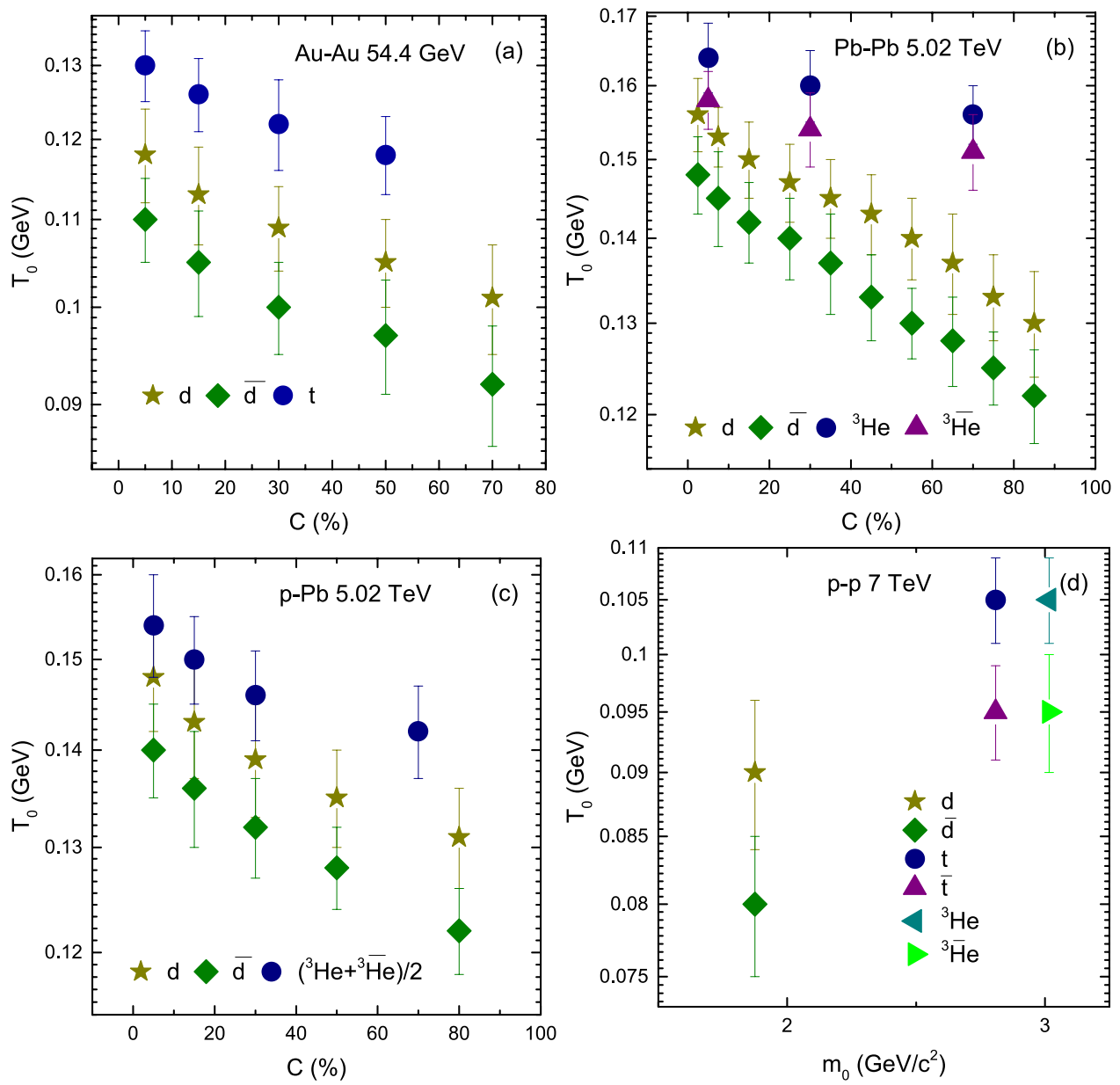

Figure 5. (a-c) Variation of $T_{0}$ with centrality and (d) variation of $T_{0}$ with $m_{0}$ for $d, \bar{d}, t, \bar{t},{ }^{3} \mathrm{He}$ and ${ }^{3} \mathrm{He}$ or $\left({ }^{3} \mathrm{He}+{ }^{3} \mathrm{He}\right) / 2$ in $\mathrm{Au}-\mathrm{Au}, \mathrm{Pb}-\mathrm{Pb}, \mathrm{p}-\mathrm{Pb}$ and $\mathrm{p}-\mathrm{p}$ collisions.

and the data are not cross-section, but they are proportional to the volumes of sources of producing various particles. Therefore it is significant to study $N_{0}$ dependence.

Figure 9 shows the variation of $T_{0}$ with $\beta_{T}$. It is observed that central collisions correspond to larger $T_{0}$ and $\beta_{T}$. The correlation between $T_{0}$ and $\beta_{T}$ is positive. This is in agreement with Ref. ${ }^{23}$ and in disagreement with Ref. ${ }^{25}$. In panel a the correlation of $T_{0}$ and $\beta_{T}$ is larger for triton and that of deuteron is larger than anti-deuteron. In panel $\mathrm{b}$ the correlation of $T_{0}$ and $\beta_{T}$ is larger for helions than deuterons and that of deuteron is larger for than anti-deuteron. Similarly in panel $c$ the helions has larger correlation between $T_{0}$ and $\beta_{T}$ than deuteron and antideuteron, and for deuteron it is larger than anti-deuteron. In general, the massive particles has larger correlation between of $T_{0}$ and $\beta_{T}$, and the particles has larger correlation than their anti-particles due to less coalescence of anti-particles.

Figure 10 shows the variation of $T_{0}$ with $V$. It is observed that central collisions correspond to larger $T_{0}$ and $V$. The correlation between $T_{0}$ and $V$ is positive. In panel a the correlation of $T_{0}$ and $V$ is larger for triton, while the correlation of $T_{0}$ and $V$ for deuterons is slightly larger than anti-deuteron. In panel $\mathrm{b}$ the correlation of $T_{0}$ and $V$ is larger for helions than deuterons, and that of helions and deuterons is slightly larger for than their anti-particles. Similarly in panel $c$ the helions has larger correlation between $T_{0}$ and $V$ than deuteron and antideuteron and deuteron has larger correlation of $T_{0}$ and $V$ than anti-deuteron. In short the massive particles has larger correlation between of $T_{0}$ and $V$, and the particles has larger correlation than their anti-particles due to less coalescence of anti-particles. 

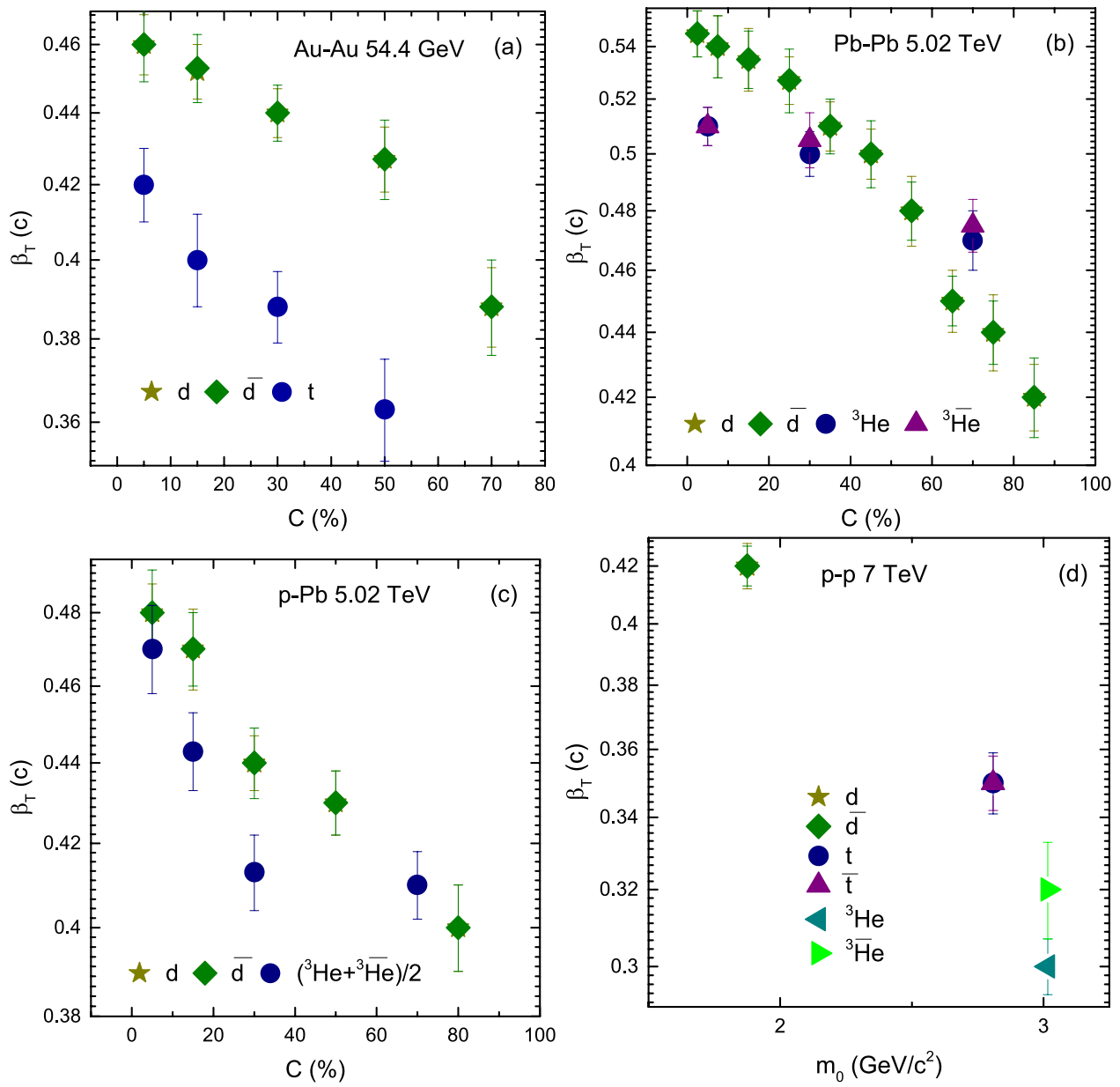

Figure 6._(a-c) Variation of $\beta_{T}$ with centrality and (d) variation of $\beta_{T}$ with $m_{0} d, \bar{d}, t, \bar{t},{ }^{3} \mathrm{He}$ and ${ }^{3} \overline{\mathrm{He}}$ or $\left({ }^{3} \mathrm{He}+{ }^{3} \mathrm{He}\right) / 2$ in $\mathrm{Au}-\mathrm{Au}, \mathrm{Pb}-\mathrm{Pb}, \mathrm{p}-\mathrm{Pb}$ and $\mathrm{p}-\mathrm{p}$ collisions.

\section{Conclusions}

The main observations and conclusions are summarized here.

(a) The transverse momentum spectra of light nuclei and their anti-nuclei produced in $\mathrm{Au}-\mathrm{Au}, \mathrm{Pb}-\mathrm{Pb}$ and $\mathrm{p}-\mathrm{Pb}$ and produced in inelastic $\mathrm{p}-\mathrm{p}$ collisions in different centrality intervals are analyzed by the BGBW model. The model results show an agreement with the experimental data in the special $p_{T}$ range measured by the STAR and ALICE Collaborations.

(b) Kinetic freezeout temperature is larger for triton and helions and their anti-particles than deuteron and anti-deuteron due to their mass. While helions and tritons have the same value of Kinetic freezeout temperature due to isospin symmetry, and dueteron, triton and helion freezeout earlier than their anti-particles respectively due to large coalescence of nucleons for the light nuclei than anti-nuclei. 

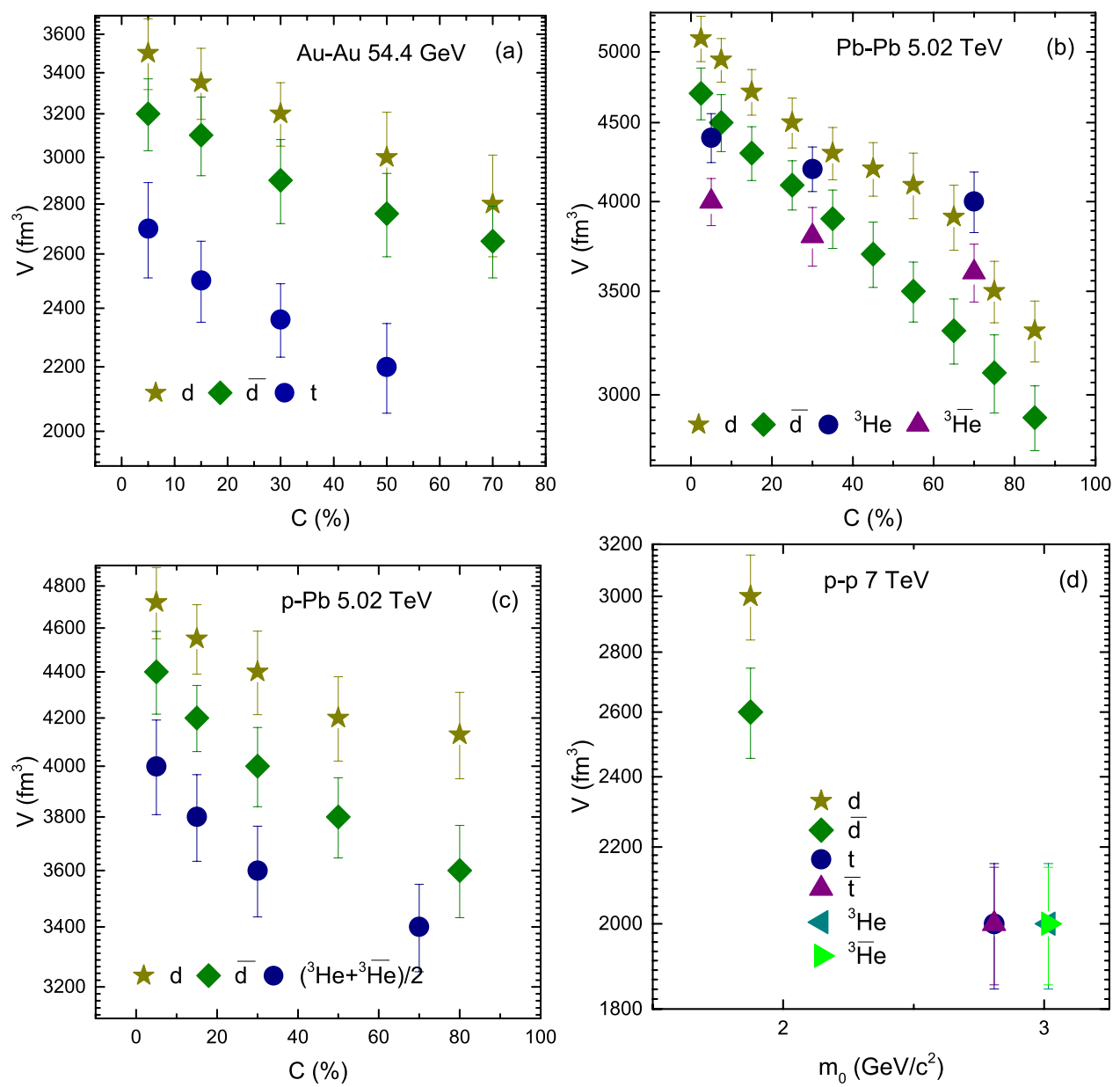

Figure 7._(a-c) Variation of $\beta_{T}$ with centrality and (d) variation of $\beta_{T}$ with $m_{0}$ for $d, \bar{d}, t, \bar{t},{ }^{3} \mathrm{He}$ and ${ }^{3} \mathrm{He}$ or $\left({ }^{3} \mathrm{He}+{ }^{3} \mathrm{He}\right) / 2$ in $\mathrm{Au}-\mathrm{Au}, \mathrm{Pb}-\mathrm{Pb}, \mathrm{p}-\mathrm{Pb}$ and $\mathrm{p}-\mathrm{p}$ collisions.

(c) Kinetic freezeout temperature decrease from central to peripheral collisions due to the decrease of participant nucleons in the peripheral collisions which lead to the decrease in the degree of excitation of the system.

(d) Transverse flow velocity increases from peripheral to central collisions due the reason that the collisions become more violent in central collisions which also expands the system rapidly.

(e) The kinetic freezeout volume decreases from central to peripheral collisions and the system reaches quickly to equilibrium state due to large number of secondary collisions by the re-scattering of partons in central collisions that decreases towards periphery. In addition, The normalization constant is larger in central collisions than in peripheral collisions. 

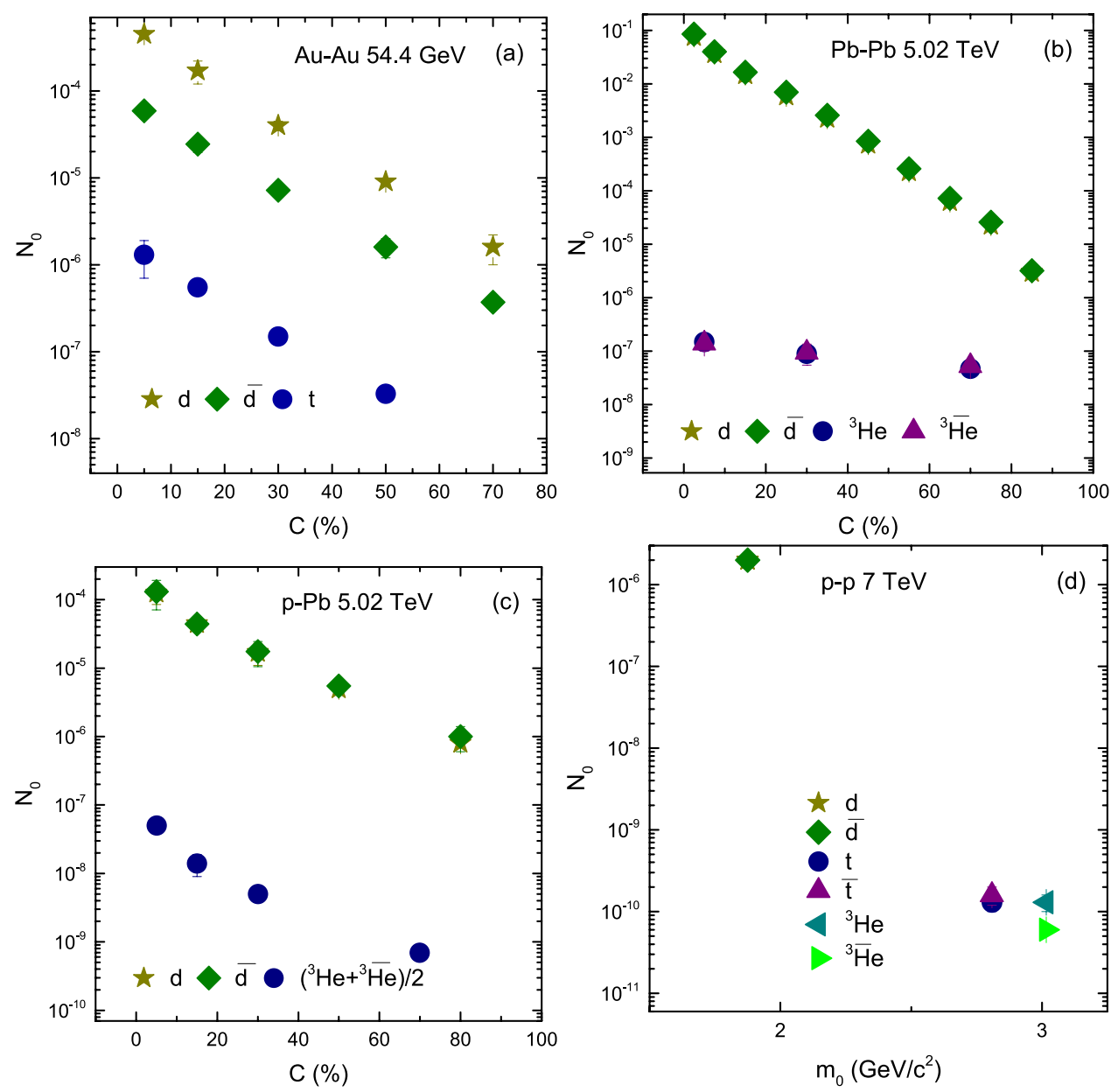

Figure 8. (a-c) Dependence of $N_{0}$ on centrality and (d) Dependence of $N_{0}$ on $m_{0}$, for $d, \bar{d}, t, \bar{t},{ }^{3} \mathrm{He}$ and ${ }^{3} \overline{\mathrm{H}}$ or $\left({ }^{3} \mathrm{He}+{ }^{3} \mathrm{He}\right) / 2$ in $\mathrm{Au}-\mathrm{Au}, \mathrm{Pb}-\mathrm{Pb}, \mathrm{p}-\mathrm{Pb}$ and $\mathrm{p}-\mathrm{p}$ collisions. 


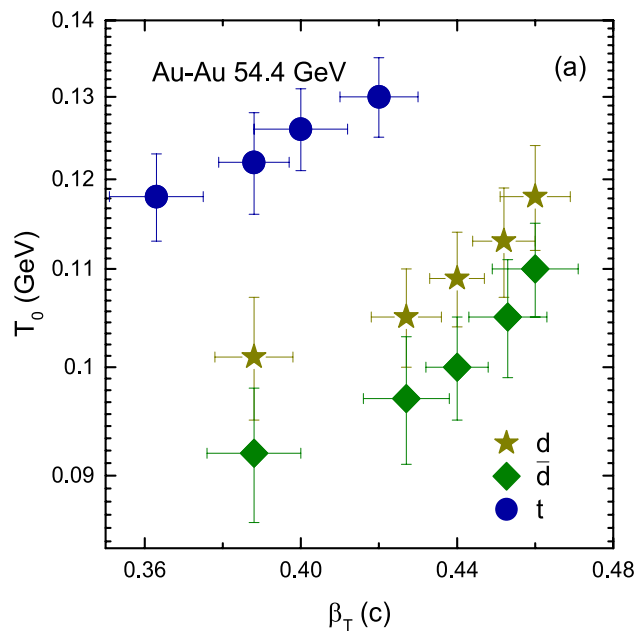

$\beta_{\mathrm{T}}(\mathrm{c})$

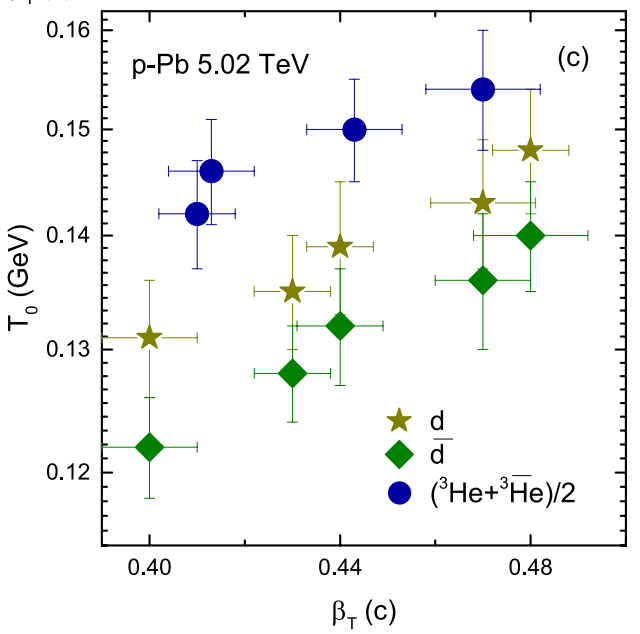

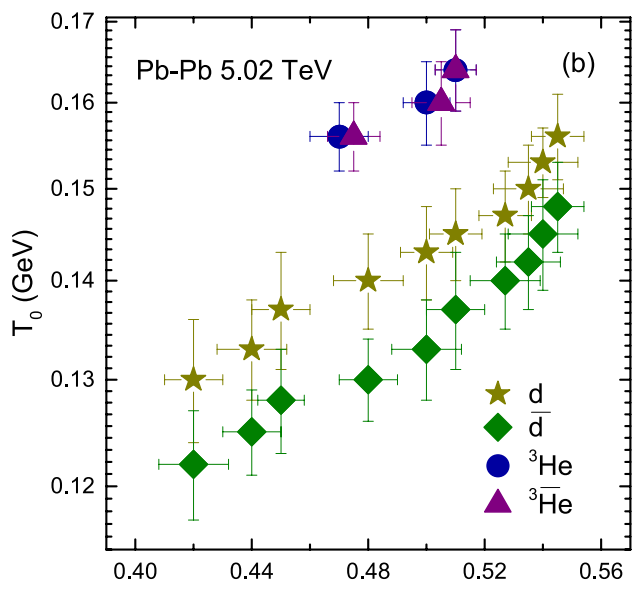

$\beta_{\mathrm{T}}(\mathrm{c})$

(b) 


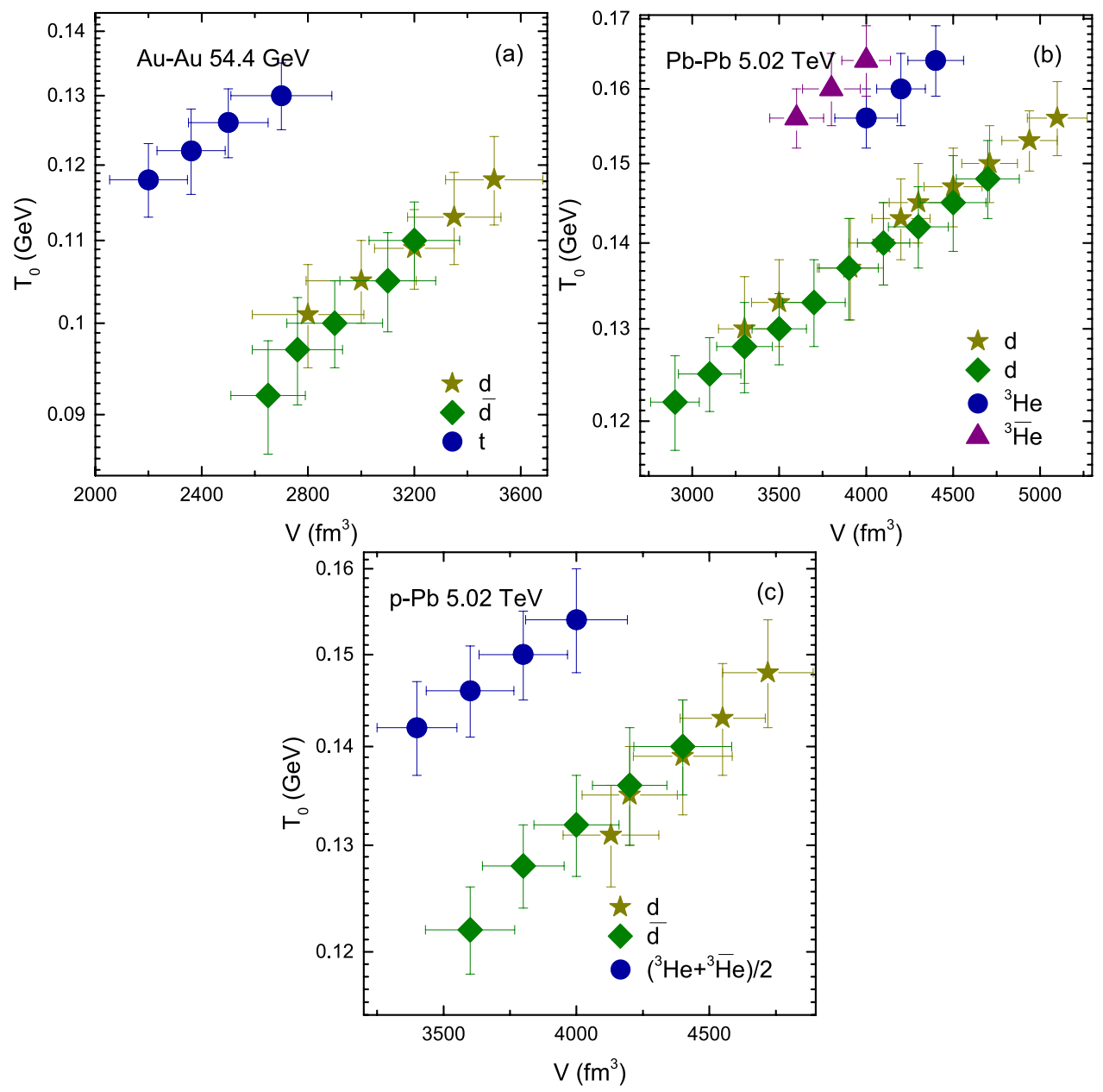

Figure 10. Variation of $T_{0}$ with $V$.

\section{Data availability}

The data used to support the findings of this study are included within the article and are cited at relevant places within the text as references.

Received: 13 April 2021; Accepted: 19 August 2021

Published online: 12 October 2021

\section{References}

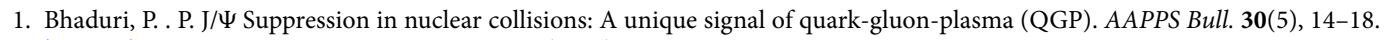
https://doi.org/10.22661/AAPPSBL.2020.30.5.1 (2020).

2. Niida, T. \& Miake, Y. Signatures of QGP at RHIC and the LHC. AAPPS Bull. 31(1), 12. https://doi.org/10.1007/s43673-021-00014-3 (2021).

3. Brambilla, N. et al. Heavy quarkonium: Progress, puzzles, and opportunities. Eur. Phys. J. C 71, 1534 (2011).

4. Schukraft, J. Heavy ion physics at the large Hadron collider: What is new? What is next? Phys. Scrip. 158, 014003 (2013).

5. Andronic, A. et al. Heavy-flavour and quarkonium production in the LHC era: From protonproton to heavy-ion collisions. Eur. Phys. J. C 76, 107 (2016).

6. Alessandro, B. et al. [NA50 Collaboration], a new measurement of J/suppression in $\mathrm{PbPb}$ collisions at $158-\mathrm{GeV}$ per nucleon. Eur. Phys. J. C 39, 335 (2005)

7. Arnaldi, R. et al. [NA60 Collaboration], production in indium-indium collisions at 158-GeV/nucleon. Phys. Rev. Lett. 99, 132302 (2007).

8. Chatterjee, S. et al. Freeze-out parameters in heavy-ion collisions at AGS, SPS, RHIC, and LHC energies. Adv. High Energy Phys. 2015, 349013. https://doi.org/10.1155/2015/349013 (2015).

9. Xu, N. [STAR], an overview of STAR experimental results. Nucl. Phys. A 931, 1-12. https://doi.org/10.1016/j.nuclphysa.2014.10. $022(2014)$

10. Chatterjee, S. \& Mohanty, B. Production of light nuclei in heavy ion collisions within multiple freezeout scenario. Phys. Rev. C 90, 034908. https://doi.org/10.1103/PhysRevC.90.034908 (2014).

11. Chatterjee, S., Mohanty, B. \& Singh, R. Freezeout hypersurface at energies available at the CERN Large Hadron Collider from particle spectra: Flavor and centrality dependence. Phys. Rev. C 92, 024917. https://doi.org/10.1103/PhysRevC.92.024917 (2015).

12. Asänen, S. S. R. [ALICE], ALICE overview. EPJ Web Conf. 126, 02026. https://doi.org/10.1051/epjconf/201612602026 (2016). 
13. Das, S., Mishra, D., Chatterjee, S. \& Mohanty, B. Freeze-out conditions in proton-proton collisions at the highest energies available at the BNL relativistic heavy ion collider and the CERN large hadron collider. Phys. Rev. C 95, 014912. https://doi.org/10.1103/ PhysRevC.95.014912 (2017).

14. Floris, M. Hadron yields and the phase diagram of strongly interacting matter. Nucl. Phys. A 931, 103-112. https://doi.org/10. 1016/j.nuclphysa.2014.09.002 (2014).

15. Huovinen, P. Chemical freeze-out temperature in hydrodynamical description of Au+Au collisions at $s(\mathrm{NN})^{* *}(1 / 2)=200-\mathrm{GeV}$. Eur. Phys. J. A 37, 121-128. https://doi.org/10.1140/epja/i2007-10611-3 (2008).

16. Andronic, A. An overview of the experimental study of quark-gluon matter in high-energy nucleus-nucleus collisions. Int. J. Mod. Phys. A 29, 1430047. https://doi.org/10.1142/S0217751X14300476 (2014).

17. De, B. Non-extensive statistics and understanding particle production and kinetic freeze-out process from $\mathrm{p}_{T}$-spectra at $2.76 \mathrm{TeV}$. Eur. Phys. J. A 50, 138. https://doi.org/10.1140/epja/i2014-14138-2 (2014).

18. Lao, H. L., Liu, F. H. \& Lacey, R. A. Extracting kinetic freeze-out temperature and radial flow velocity from an improved Tsallis distribution. Eur. Phys. J. A 53, 44 (2017).

19. Wang, Q. \& Liu, F. H. Initial and final state temperatures of antiproton emission sources in high energy collisions. Int. J. Theor. Phys. 58, 4119-4138. https://doi.org/10.1007/s10773-019-04278-2 (2019).

20. Waqas, M., Peng, G. X. \& Liu, F. H. An evidence of triple kinetic freezeout scenario observed in all centrality intervals in $\mathrm{Cu}-\mathrm{Cu}$, $\mathrm{Au}-\mathrm{Au}$ and $\mathrm{Pb}-\mathrm{Pb}$ collisions at high energies. J. Phys. G. https://doi.org/10.1088/1361-6471 (2021).

21. Waqas, M., Liu, F. H. \& Wazir, Z. Dependence of temperatures and kinetic freeze-out volume on centrality in $\mathrm{Au}-\mathrm{Au}$ and $\mathrm{Pb}-\mathrm{Pb}$ collisions at high energy. Adv. High Energy Phys. 2020, 8198126. https://doi.org/10.1155/2020/8198126 (2020).

22. Waqas, M., Liu, F. H., Fakhraddin, S. \& Rahim, M. A. Possible scenarios for single, double, or multiple kinetic freeze-out in high energy collisions. J. Phys. 93, 1329-1343. https://doi.org/10.1007/s12648-019-01396-9 (2019).

23. Waqas, M. \& Li, B. C. Kinetic freeze-out temperature and transverse flow velocity in Au-Au collisions at RHIC-BES energies. $A d v$. High Energy Phys. 2020, 1787183 (2020).

24. Che, G. R., Gu, J. B., Zhang, W. C. \& Zheng, H. Identified particle spectra in $\mathrm{Pb}-\mathrm{Pb}$ and $\mathrm{p}-\mathrm{Pb}$ collisions with a modified Tsallis blast-wave model. http://arxiv.org/abs/2010.14880.

25. Adamczyk, L. et al. [STAR], bulk properties of the medium produced in relativistic heavy-ion collisions from the beam energy scan program. Phys. Rev. C 96, 044904. https://doi.org/10.1103/PhysRevC.96.044904 (2017).

26. Uddin, S., Bhat, R. A. \& Bashir, I. U. Study of centrality dependence of transverse momentum spectra of hadrons and the freeze-out parameters at root (sNN) of $62.4 \mathrm{GeV}, 130 \mathrm{GeV}$ and $200 \mathrm{GeV}$. http://arxiv.org/abs/1412.2663.

27. Bashir, I. . u \& Uddin, S. Centrality dependence of $K^{*}(892)^{0}$ and $\phi(1020)$ production at LHC. Commun. Theor. Phys 68, 500. https:// doi.org/10.1088/0253-6102/68/4/500 (2017).

28. Khuntia, A., Sharma, H., Kumar Tiwari, S., Sahoo, R. \& Cleymans, J. Radial flow and differential freeze-out in proton-proton collisions at $\sqrt{s}=7 \mathrm{TeV}$ at the LHC. Eur. Phys. J. A 55, 3. https://doi.org/10.1140/epja/i2019-12669-6 (2019).

29. Lao, H. . L., Wei, H. . R., Liu, F. . H. \& Lacey, R. . A. An evidence of mass-dependent differential kinetic freeze-out scenario observed in Pb-Pb collisions at 2.76 TeV. Eur. Phys. J. A 52, 203. https://doi.org/10.1140/epja/i2016-16203-2 (2016).

30. Thakur, D., Tripathy, S., Garg, P., Sahoo, R. \& Cleymans, J. Indication of differential kinetic freeze-out at RHIC and LHC energies. Acta Phys. Polon. Suppl. 9, 329. https://doi.org/10.5506/APhysPolBSupp.9.329 (2016).

31. Shao, M. et al. Examine the species and beam-energy dependence of particle spectra using Tsallis Statistics. J. Phys. G 37, 085104. https://doi.org/10.1088/0954-3899/37/8/085104 (2010).

32. Tawfik, A. N. Equilibrium statistical-thermal models in high-energy physics. Int. J. Mod. Phys. A 29, 1430021. https://doi.org/10. 1142/S0217751X1430021X (2014).

33. Abelev, B. I. et al. [STAR], systematic measurements of identified particle spectra in $p p, d^{+} \mathrm{Au}$ and Au+Au collisions from STAR. Phys. Rev. C 79, 034909. https://doi.org/10.1103/PhysRevC.79.034909 (2009).

34. Schnedermann, E., Sollfrank, J. \& Heinz, U. W. Thermal phenomenology of hadrons from 200-A/GeV S+S collisions. Phys. Rev. C 48, 2462-2475. https://doi.org/10.1103/PhysRevC.48.2462 (1993).

35. Abelev, B. I. et al. [STAR], identified particle production, azimuthal anisotropy, and interferometry measurements in $\mathrm{Au}+\mathrm{Au}$ collisions at $\mathrm{s}(\mathrm{NN})^{* *}(1 / 2)=9.2-\mathrm{GeV}$. Phys. Rev. C 81, 024911. https://doi.org/10.1103/PhysRevC.81.024911 (2010).

36. Tang, Z. et al. Spectra and radial flow at RHIC with Tsallis statistics in a blast-wave description. Phys. Rev. C 79, 051901. https:// doi.org/10.1103/PhysRevC.79.051901 (2009).

37. Russo, $\mathrm{R}$. Measurement of $\mathrm{D}^{+}$meson production in $\mathrm{p}-\mathrm{Pb}$ collisions with the ALICE detector. http://arxiv.org/abs/1511.04380.

38. Takeuchi, S., Murase, K., Hirano, T., Huovinen, P. \& Nara, Y. Effects of hadronic rescattering on multistrange hadrons in highenergy nuclear collisions. Phys. Rev. C 92, 044907. https://doi.org/10.1103/PhysRevC.92.044907 (2015).

39. Heinz, U. W. Lecture Notes for Lectures Presented at the 2nd CERNLatin-American School of High-Energy Physics (1C14June 2003. http://arxiv.org/abs/hep-ph/0407360 (2004).

40. Heiselberg, H. \& Levy, A. M. Elliptic flow and HBT in noncentral nuclear collisions. Phys. Rev. C 59, 2716-2727. https://doi.org/ 10.1103/PhysRevC.59.2716 (1999).

41. Wei, H. R., Liu, F. H. \& Lacey, R. A. Kinetic freeze-out temperature and flow velocity extracted from transverse momentum spectra of final-state light flavor particles produced in collisions at RHIC and LHC. Eur. Phys. J. A 52, 102. https://doi.org/10.1140/epja/ i2016-16102-6 (2016).

42. Wei, H. R., Liu, F. H. \& Lacey, R. A. Disentangling random thermal motion of particles and collective expansion of source from transverse momentum spectra in high energy collisions. J. Phys. G 43, 125102. https://doi.org/10.1088/0954-3899/43/12/125102 (2016).

43. Cleymans, J. \& Worku, D. Relativistic thermodynamics: Transverse momentum distributions in high-energy physics. Eur. Phys. J. A 48, 160. https://doi.org/10.1140/epja/i2012-12160-0 (2012).

44. Zheng, H. \& Zhu, L. Comparing the Tsallis distribution with and without thermodynamical description in $p+p$ collisions. Adv. High Energy Phys. 2016, 9632126. https://doi.org/10.1155/2016/9632126 (2016).

45. Lao, H. . L., Liu, F. . H., Li, B. . C. \& Duan, M. . Y. Kinetic freeze-out temperatures in central and peripheral collisions: Which one is larger? Nucl. Sci. Technol. 29, 82. https://doi.org/10.1007/s41365-018-0425-x (2018).

46. Zhang, S., Ma, Y. G., Chen, J. H. \& Zhong, C. Production of Kaon and $\Lambda$ in nucleus-nucleus collisions at ultrarelativistic energy from a blast-wave model. Adv. High Energy Phys. 2015, 460590. https://doi.org/10.1155/2015/460590 (2015).

47. Bialas, A., Florkowski, W. \& Zalewski, K. Blast-wave model description of the Hanbury-Brown-Twiss radii in pp collisions at LHC energies. J. Phys. G 42, 045001. https://doi.org/10.1088/0954-3899/42/4/045001 (2015).

48. Sun, X., Masui, H., Poskanzer, A. . M. \& Schmah, A. Blast wave fits to elliptic flow data at $\sqrt{s_{\mathrm{NN}}}=7.7-2760 \mathrm{GeV}$. Phys. Rev. C 91, 024903. https://doi.org/10.1103/PhysRevC.91.024903 (2015).

49. Florkowski, W. Blast-wave model description of the HBT radii measured in $p p$ collisions at the LHC energies. Acta Phys. Polon. $B$ 47, 2241. https://doi.org/10.5506/APhysPolB.47.2241 (2016).

50. Cimerman, J., Tomasik, B., Csanad, M. \& Lokos, S. Higher-order anisotropies in the blast-wave model-Disentangling flow and density field anisotropies. Eur. Phys. J. A 53, 161. https://doi.org/10.1140/epja/i2017-12349-7 (2017).

51. Arnison, G. et al. [UA1], transverse momentum spectra for charged particles at the CERN proton anti-proton collider. Phys. Lett. B 118, 167-172. https://doi.org/10.1016/0370-2693(82)90623-2 (1982). 
52. Odorico, R. Does a transverse energy trigger actually trigger on large $\mathrm{p}(\mathrm{t})$ jets? Phys. Lett. B 118, 151-154. https://doi.org/10.1016/ 0370-2693(82)90620-7 (1982).

53. Biyajima, M., Mizoguchi, T. \& Suzuki, N. Analyses of whole transverse momentum distributions in $p \bar{p}$ and $p p$ collisions by using a modified version of Hagedorn's formula. Int. J. Mod. Phys. A 32, 1750057. https://doi.org/10.1142/S0217751X17500579 (2017).

54. Abelev, B. B. et al. [ALICE], production of $\Sigma(1385)^{ \pm}$and $\Xi(1530)^{0}$ in proton-proton collisions at $\sqrt{s}=7$ TeV. Eur. Phys. J. C 75, 1. https://doi.org/10.1140/epjc/s10052-014-3191-x (2015).

55. Hagedorn, R. Riv. Nuovo Cimento 6, 1-50. https://doi.org/10.1007\%2FBF02740917 (1983)

56. Zhang, D. [STAR], light nuclei $(d, t)$ production in Au + Au collisions at $\sqrt{s_{N N}}=77-200 \mathrm{GeV}$. Nucl. Phys. A 1005, 121825. https:// doi.org/10.1016/j.nuclphysa.2020.121825 (2021).

57. Puccio, M. Study of the production of nuclei and anti-nuclei at the LHC with the ALICE experiment. CERN-THESIS-2017-338.

58. Acharya, S. et al. [ALICE], multiplicity dependence of light (anti-)nuclei production in $\mathrm{p}-\mathrm{Pb}$ collisions at $\sqrt{s_{\mathrm{NN}}}=5.02 \mathrm{TeV}$. Phys. Lett. B 800, 135043. https://doi.org/10.1016/j.physletb.2019.135043 (2020).

59. Acharya, S. et al. [ALICE], production of (anti-) $)^{3} \mathrm{He}$ and (anti-) ${ }^{3} \mathrm{H}$ in p-Pb collisions at $\sqrt{s_{\mathrm{NN}}}=5.02 \mathrm{TeV}$. Phys. Rev. C 101, 044906 . https://doi.org/10.1103/PhysRevC.101.044906 (2020).

60. Acharya, S. et al. [ALICE], production of deuterons, tritons, ${ }^{3} \mathrm{He}$ nuclei and their antinuclei in $\mathrm{p}-\mathrm{p}$ collisions at $\sqrt{\mathbf{s}}=0.9,2.76$ and 7 TeV. Phys. Rev. C 97, 024615. https://doi.org/10.1103/PhysRevC.97.024615 (2018).

\section{Acknowledgements}

The authors would like to thank support from the National Natural Science Foundation of China (Grant Nos. 11875052,11575190 , and 11135011).

\section{Author contributions}

The authors contributed to the paper in this way: conceptualization, M.W.;methodology, M.W. and G.X.P., F.H.L.; software, M.W.; validation, M.W. and Z.W.; formalanalysis, M.W.; investigation, M.W., F.H.L.; resources,G.X.P.; data curation, M.W., Z.W.; writing C originaldraft preparation, M.W., Z.W.; writing C review and editing,M.W., F.H.L. and G. X.P.; visualization, M.W.; supervision,G.X.P.; project administration, G.X.P.; fundingacquisition, G.X.P.

\section{Competing interests}

The authors declare no competing interests.

\section{Additional information}

Correspondence and requests for materials should be addressed to G.X.P.

Reprints and permissions information is available at www.nature.com/reprints.

Publisher's note Springer Nature remains neutral with regard to jurisdictional claims in published maps and institutional affiliations.

(c) (i) Open Access This article is licensed under a Creative Commons Attribution 4.0 International License, which permits use, sharing, adaptation, distribution and reproduction in any medium or format, as long as you give appropriate credit to the original author(s) and the source, provide a link to the Creative Commons licence, and indicate if changes were made. The images or other third party material in this article are included in the article's Creative Commons licence, unless indicated otherwise in a credit line to the material. If material is not included in the article's Creative Commons licence and your intended use is not permitted by statutory regulation or exceeds the permitted use, you will need to obtain permission directly from the copyright holder. To view a copy of this licence, visit http://creativecommons.org/licenses/by/4.0/.

(C) The Author(s) 2021 